Universidade Tecnológica Federal do Paraná - UTFPR

Campus Ponta Grossa - Paraná - Brasil

ISSN 1808-0448/v. 04, n. 01: p. 39-56, 2008

D.O.I.: $10.3895 / \mathrm{S} 1808-04482008000100003$

\section{Revista Gestão Industrial}

\title{
INCERTEZA DE PORTFÓLIO DE PROJETOS
}

\section{UNCERTAINTY OF PROJECT'S PORTFOLIO}

\author{
Renato de Oliveira Moraes ${ }^{1}$; Fernando José Barbin Laurindo ${ }^{2}$; Priscila Santiago Pereira ${ }^{3}$ \\ ${ }^{1}$ Fead-Minas - Belo Horizonte - Brasil renato.moraes@,fead.br \\ ${ }^{2}$ Universidade de São Paulo - USP - São Paulo - Brasil fjblau@usp.br \\ ${ }^{3}$ Universidade Federal de Minas Gerais - UFMG - Belo Horizonte - Brasil prisicla.santiago@yahoo.com.br
}

\begin{abstract}
Resumo
Os métodos de composição de carteira de projetos costumam destacar a importância da incerteza como variável do processo. Em geral, a avaliação da incerteza é feita para os projetos de forma individual. Tanto no que se refere à incerteza em relação aos recursos necessários como também à incerteza associada aos resultados a serem alcançados. O gerente dos gerentes de projetos é responsável por todos os projetos (e seus gerentes) e está disposto a assumir (e até conta) com algum grau de insucessos individuais nos projetos sob sua responsabilidade, mas seu interesse final é no resultado global do conjunto de projetos em desenvolvimento. Assim, na construção de uma carteira de projetos é muito importante uma avaliação da incerteza das alternativas possíveis da carteira de desenvolvimento - incerteza associada a um conjunto particular de projetos. Este artigo retoma a proposta de Moraes e Laurindo (2004) para composição de carteira de desenvolvimento ampliando o tratamento dado à incerteza dos projetos da carteira. $O$ uso de Fatores Críticos de Sucesso - FCS (ROCKART, 1979) é substituído pelo Modelo de Gaps (SLACK et all, 1996). Além disto, a estimativa do impacto de cada projeto sob os indicadores de desempenho organizacional é feita através de três cenários distintos: pessimista, mais provável, e otimista. A avaliação das diferentes combinações possíveis entre os projetos permite avaliar a probabilidade dos diferentes impactos que a carteira pode ter sobre os indicadores de desempenho organizacional.
\end{abstract}

Palavras-chave: incerteza de projetos, incerteza de portfolio, gestão de portfolio

\section{Introdução}

Este artigo retoma a proposta de Moraes e Laurindo (2004) para composição de carteira de desenvolvimento ampliando o tratamento dado a incerteza dos projetos da carteira. $\mathrm{O}$ texto procura ser inespecífico em relação a natureza dos projetos da carteira. Por trás disto está a percepção e o desejo que a proposta seja útil a diferentes tipos de projetos.

Os métodos de composição de carteira de projetos costumam destacar a importância da incerteza como variável do processo. Em geral, a avaliação da incerteza é feita para os projetos de 
forma individual. Tanto no que se refere à incerteza em relação aos recursos necessários como também à incerteza associada aos resultados a serem alcançados.

Os resultados esperados de um projeto são quantificados em relação aos benefícios monetários gerados ou em função do impacto e do alinhamento à estratégia organizacional. Apesar dos resultados econômico-financeiros serem de importância inegável, nem sempre os benefícios dos projetos se manifestam de forma tão objetiva e tangível.

Assim, a avaliação dos benefícios por meio da estimativa de impacto ou de alinhamento à estratégia organizacional costuma ser bastante necessária.

Para um gerente de projeto a avaliação da incerteza (e dos riscos) associada aos seus projetos é algo fundamental, mas para o gerente dos gerentes de projetos esta informação não é suficiente. Ele é responsável por todos os projetos (e seus gerentes) e está disposto a assumir (e até conta) com algum grau de insucessos individuais nos projetos sob sua responsabilidade, mas seu interesse final é no resultado global do conjunto de projetos em desenvolvimento. Assim, na construção de uma carteira de projetos de desenvolvimento é muito importante uma avaliação da incerteza das alternativas possíveis da carteira de desenvolvimento - incerteza associada a um conjunto particular de projetos de desenvolvimento.

Este artigo apresenta um procedimento para a avaliação da incerteza de uma carteira em relação à sua contribuição para os fatores críticos de sucesso (FCS) da organização. Isto é feito a partir de uma estimativa da incerteza associada a contribuição de cada projeto aos FCS. Esta estimativa é feita através de três cenários distintos:

- pessimista: refere-se ao pior caso possível, onde seriam obtidos os resultados mínimos possíveis com o projeto;

- mais provável: refere-se a situação que tem mais chances de ocorrer e seus impactos sobre os FCS;

- otimista: refere-se ao melhor caso possível, onde os impactos mais significativos sobre os FCS seriam obtidos.

A avaliação das diferentes combinações possíveis entre os projetos permite avaliar a probabilidade dos diferentes impactos que a carteira pode ter sobre os FCS. Assim, para uma determinada carteira de projetos pode ser identificado um perfil da sua contribuição para cada FCS. Este perfil é caracterizado através 5 parâmetros:

- Mínimo: refere-se ao impacto mínimo sobre um determinado FCS, equivale a improvável situação na qual todos os projetos terminam da forma pessimista; 
- Primeiro quartil: a probabilidade de que o impacto sobre um determinado FCS seja menor ou igual a este valor é $25 \%$;

- Mediana: a probabilidade de que o impacto sobre um determinado FCS seja menor ou igual a este valor é 50\% (esta também é a probabilidade do impacto ser igual ou maior que este valor);

- Terceiro quartil: a probabilidade de que o impacto sobre um determinado FCS seja maior ou igual a este valor é $25 \%$;

- Máximo: refere-se ao impacto máximo sobre um determinado FCS, equivale a improvável situação na qual todos os projetos terminam da forma otimista.

O artigo apresenta uma revisão do problema de composição de carteira de projetos, com especial atenção aos modelos que incorporam a variável incerteza no processo de decisão. Em seguida é descrito o procedimento de análise da incerteza da carteira a partir da incerteza dos projetos que a compõe ilustrado por um pequeno caso.

\section{Incerteza de Projetos em Portfólio de Projetos}

Apesar de serem conceitos relacionados, existe uma distinção entre risco e incerteza de projetos. Incerteza refere-se, geralmente, ao desconhecimento dos possíveis desdobramentos de uma decisão ou linha de conduta. A idéia de risco, por sua vez, inclui uma quantificação das probabilidades de ocorrência de eventos futuros e de suas possíveis conseqüências. Em particular seus impactos nas metas dos projetos. Apesar de reconhecer esta diferença, de certa forma, mistura estes conceitos na medida em que tenta realizar uma quantificação das incertezas associadas a um conjunto de projetos.

A incerteza da carteira de projetos é, obviamente, resultado direto da incerteza dos projetos que a compõe. Assim a incerteza dos projetos e a relação entre eles, em termos de uso compartilhado de recursos, tecnologias e competências comuns e de outras formas de sinergia, estabelece limites e condiciona a incerteza da carteira de projetos.

A incerteza dos projetos é um elemento freqüentemente considerado nos modelos de composição de portfólio bem como nos modelos de gestão de projetos em geral.

O que se procura aqui é, a partir de uma avaliação da incerteza dos projetos em relação aos seus resultados, chegar a uma medida da incerteza do portfólio em relação aos seus resultados. Esta incerteza em relação aos resultados refere-se ao atendimento das necessidades que motivaram a 
realização dos projetos.

Esta incerteza em relação aos resultados, que tem uma natureza e escopo mais específicos, depende da incerteza e da complexidade dos projetos num sentido amplo e que são tratados a seguir.

A elaboração da carteira, de modo geral, considera uma série de fatores. Dentre eles a incerteza de cada projeto. A avaliação da incerteza individual associada a cada projeto pode aparecer nas várias etapas da avaliação ex ante - inspeção (screaming), avaliação e seleção (SOUDER, 1983), e assume formas de gráficos, listas de verificação, modelos de pesos (ranking), etc.

Diferentes visões para incerteza (e de suas dimensões) dos projetos podem ser encontradas na literatura. Moraes (1999) trata a incerteza como a diferença entre as informações necessárias e as disponíveis para execução do projeto, e a decompõe em:

1. Incerteza quanto aos resultados de curto prazo esperados do projeto em termos de:

- Custo;

- Prazo;

- Qualidade.

2. Incerteza em relação às atividades a serem executadas;

3. Incerteza em relação à duração das atividades do projeto;

4. Incerteza quanto às relações de precedência entre as atividades;

5. Incerteza em relação ao custo do projeto;

6. Incerteza em relação à disponibilidade financeira para execução do projeto;

7. Incerteza em relação à tecnologia e ao domínio das competências necessárias ao projeto;

8. Incerteza em relação à disponibilidade de recursos (material e humano) para execução do projeto.

Segundo Archibald (1976), a incerteza de um projeto diminui à medida que ele avança, conforme mostra a figura 1. Isto ilustra porque projetos já iniciados e mais próximos de seu final, apresentam um risco menor.

Figura 1. Incerteza versus fase do projeto 

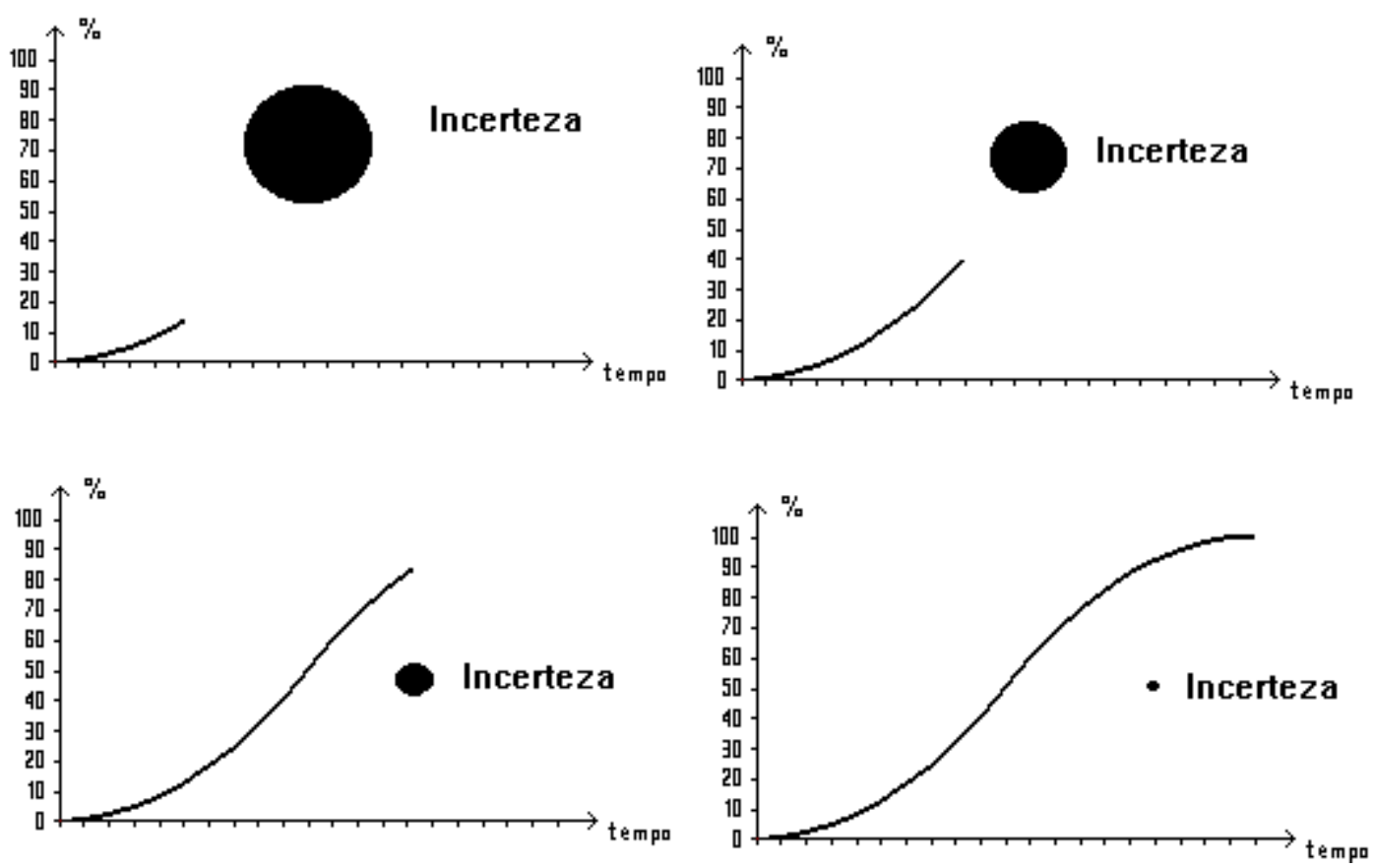

Fonte: Archibald (1976)

Shenhar et al (2002) abordam, especificamente, a incerteza tecnológica dos projetos e a classifica em quatro categorias:

- Projetos de tecnologia baixa (Low-tech projects);

- Projetos de tecnologia média (Medium-tech projects);

- Projetos de alta tecnologia (High-tech projects);

- Projetos de tecnologia de ponta (Super high-tech projects).

Projetos de tecnologia baixa (Low-tech projects): baseados em tecnologias bem estabelecidas nas quais todos os atores do setor têm igual competência. Embora este tipo de projeto possa ter um grande tamanho, nenhuma nova tecnologia nova é gerada ou adquirida em nenhum momento do projeto. A incerteza tecnológica é, em termos práticos, nula. A maioria dos projetos de construção civil está nesta categoria

Projetos de tecnologia média (Medium-tech projects): usam principalmente tecnologias existentes mas, de alguma forma, incorporam alguma nova tecnologia ou nova característica. Tais projetos são caracterizados por um nível de incerteza tecnológica relativamente baixa. A nova tecnologia, ou nova característica, é geralmente uma vantagem do projeto. Projetos industriais de inovação incremental de processo e de produtos são exemplos deste tipo de projeto.

Projetos de alta tecnologia (High-tech projects): usa principalmente tecnologias que são novas, mas que foram desenvolvidas, pelo menos parcialmente, antes do início do projeto. $\mathrm{O}$ uso integrado de diferentes tecnologias pela primeira vez faz com a incerteza tecnológica destes 
projetos seja elevada. Projetos de defesa que fazem uso de tecnologias novas, mas que já foram desenvolvidas anteriormente, são exemplos deste tipo de projeto.

Projetos de tecnologia de ponta (Super high-tech projects): são baseados em novas tecnologias que podem não estar disponíveis no início do projeto. Estas tecnologias ainda estão em fase experimental ou ainda serão desenvolvidas durante a execução do projeto. Estes projetos possuem uma elevada incerteza tecnológica e são relativamente raros, envolvem alto risco, e são, geralmente, realizados por grandes organizações. Um exemplo desta categoria é o projeto Apolo (foguete lunar).

A incerteza de projetos está também relacionada ao tipo de projeto. Moraes e Laurindo (2004) utilizam uma tipologia de projetos de sistemas da informação (SI) com as seguintes categorias:

- Obrigatórios: projetos que não possuem alternativas à sua execução. Esse foi o caso, por exemplo, das aplicações que tinham problemas relacionados ao bug do milênio, já que as empresas eram obrigadas a modificar seus SI para evitar sérios problemas futuros. Alterações legais podem ter o mesmo efeito sobre os projetos de SI;

- Infra-estrutura: nesse tipo de projeto os benefícios imediatos são pouco significativos, mas criam novas e importantes oportunidades. Exemplos dessa categoria são: implantação de um novo banco de dados corporativo, de uma rede local, implantação de intranets ou uma nova estrutura organizacional;

- Incremental: projetos que envolvem tecnologia ou processo de negócio bem conhecido, cujos impactos são facilmente previstos. Trazem ganhos incrementais em eficiência e/ou eficácia;

- Exploratórios: projetos que envolvem novas tecnologias (em relação ao conhecimento dominado pela organização), novas estratégias de negócio e/ou novas estruturas/processos organizacionais. São projetos de grande incerteza intrínseca, contudo, se forem bemsucedidos, podem trazer grandes ganhos (em termos de eficiência e/ou eficácia) para a organização.

Projetos obrigatórios, pela sua natureza, devem ser concebidos com uma baixíssima incerteza, já que as conseqüências de fracasso tendem a ser muito ruins. Projetos de infra-estrutura e incrementais tendem a ter uma incerteza maior que os obrigatórios porém significativamente menor que os projetos exploratórios. Assim, a construção de uma carteira equilibrada em termos de custos e benefícios esperados deve considerar também um correto balanceamento entre os tipos de projetos 
dentro da carteira de desenvolvimento.

Outra característica dos projetos que afeta sua probabilidade de sucesso (ou a incerteza em relação ao seu impacto) é a complexidade do projeto. Segundo Bacarrini (1996) a complexidade pode ter duas interpretações diferentes (mas não excludentes):

- Diferenciação ou diversificação, que considera a quantidade de tipos de elementos distintos existentes no projeto. Um projeto pode ser chamado de complexo quando a diversidade destes elementos é grande;

- Interdependência, que é a relação ou interação entre os elementos do projeto. Quando os elementos possuem uma grande interdependência, o projeto pode ser denominado de complexo.

A complexidade de um projeto pode estar relacionada tanto com as suas características técnicas como com as organizacionais. Existem dois tipos de complexidade, a organizacional e a tecnológica. Como ilustra o quadro 1, esta complexidade pode ser dividida em quatro categorias.

Quadro 1: Complexidade de projetos

\begin{tabular}{|c|c|c|}
\hline & Organizacional & Tecnológica \\
\hline Diferenciação & $\begin{array}{l}\text { A estrutura organizacional complexa } \\
\text { possui partes diferentes } \\
\text { a. Diferenciação vertical: número de } \\
\text { níveis administrativos; } \\
\text { b. Diferenciação horizontal: } \\
\text { 1. unidades organizacionais: } \\
\text { departamentos grupos } \\
\text { 2. estrutura das atividades: } \\
\text { • divisão do trabalho: as tarefas } \\
\text { são projetadas de forma que } \\
\text { qualquer um possa executá-las; } \\
\text { • especialização profissional: a } \\
\text { execução das tarefas está a } \\
\text { cargo de especialistas. }\end{array}$ & $\begin{array}{l}\text { Refere-se à variedade de algum } \\
\text { aspecto das atividades, como: } \\
\text { - Quantidade e diversidade de } \\
\text { inputs e/ou outputs; } \\
\text { - Quantidade de ações ou } \\
\text { atividades diferentes } \\
\text { (tecnologicamente, } \\
\text { temporalmente e/ou } \\
\text { espacialmente ) e separadas, } \\
\text { necessárias para produzir um } \\
\text { produto do projeto; } \\
\text { - Número de especialidades } \\
\text { envolvidas no projeto. }\end{array}$ \\
\hline Interdependência & $\begin{array}{l}\text { O grau de interdependência } \\
\text { operacional entre as unidades } \\
\text { organizacionais: } \\
\text { - simultâneo; } \\
\text { - seqüencial; } \\
\text { - recíproco. }\end{array}$ & $\begin{array}{l}\text { A complexidade como } \\
\text { interdependência tecnológica engloba: } \\
\text { - entre atividades; } \\
\text { - dentro de uma rede de atividades; } \\
\text { - entre tecnologias diferentes; } \\
\text { - entre inputs. } \\
\text { O grau de interdependência tenológica } \\
\text { possui os seguintes níveis: } \\
\text { - simultâneo; } \\
\text { - sequencial; } \\
\text { - recíproco. }\end{array}$ \\
\hline
\end{tabular}

Fonte: Bacarrini (1996) 


\section{Fatores Críticos de Sucesso e Modelo de GAPS's}

Uma das primeiras propostas para o problema de seleção e priorização de projetos de tecnologia da informação foi o método dos Fatores Críticos de Sucesso (FCS), proposto por Rockart (1979) como procedimento para seleção e priorização de projetos de tecnologia da informação, especialmente sistemas de informação gerenciais e executivos. Ele é baseado na identificação, por parte dos altos executivos da organização, dos elementos onde um resultado satisfatório "garante o sucesso do desempenho competitivo da organização". O autor afirma que os principais FCS podem ser identificados na estrutura do setor, na estratégia competitiva, na posição da indústria, na localização geográfica e nos fatores ambientais e temporais. Embora tenha sido concebido primariamente para definição de sistemas de informação, esse método apresenta importante impacto nas práticas gerenciais e de planejamento estratégico. É comum definir FCS para diferentes funções da empresa, como FCS de marketing, de manufatura, de projeto, dentre outros.

Slack et all (1996) apresentam um procedimento para avaliar o desempenho estratégico através da Matriz Importância x Desempenho ou Modelo de GAPs. Sob certos aspectos pode ser considerada uma evolução dos FCS de Rockart.

Aqui os indicadores de desempenho são avaliados em duas dimensões e cada dimensão tem três faixas de classificação que, por sua vez, possuem três sub-faixas (quadro 2 e figura 2).

A primeira dimensão - Importância para o cliente - destaca que os indicadores não têm importância igual dentro de um determinado posicionamento estratégico que a organização assume em seu mercado. Assim, dentro destas dimensão, os indicadores se classificam em:

- Ganhadores de pedido: são aqueles utilizados pelos clientes para escolha de seu fornecedor;

- Qualificadores: são aqueles onde os clientes exigem apenas um nível mínimo de desempenho. Caso a empresa não atinja esta patamar mínimo, provavelmente os clientes não irão considerá-la como um possível fornecedor. Além dito, se o desempenho da empresa nestes indicadores for substancialmente maior que os da concorrência, eles pouco contribuirão para que os clientes escolha a empresa como fornecedora. Um desempenho superior aqui tem um baixo impacto positivo, mas um desempenho inferior tem um alto impacto negativo;

- Menos importantes: são critérios de importância secundária e que não se encaixam em nenhuma das duas categorias anteriores.

A segunda dimensão - Desempenho em relação a concorrência - é uma avaliação por vezes 
subjetiva do desempenho da empresa em relação à concorrência. Desta forma o desempenho de um indicador não é nunca avaliado através de uma escala absoluta, sempre através de uma escala relativa (à concorrência). Assim, dentro desta dimensão, os indicadores são classificados:

- Melhor que a concorrência;

- Igual a concorrência;

- Pior que a concorrência.

Quadro 2:: Dimensões dos indicadores de desempenho de SLACK

\begin{tabular}{|l|l|l|}
\hline Dimensões & Faixas & Sub-faixas \\
\hline \multirow{2}{*}{$\begin{array}{l}\text { Importância para o } \\
\text { cliente }\end{array}$} & ganhadores de pedido & 1,2 e 3 \\
\cline { 2 - 3 } & qualificadores & 4,5 e 6 \\
\cline { 2 - 3 } & menos importantes & 7,8 e 9 \\
\hline \multirow{2}{*}{$\begin{array}{l}\text { Desempenho em } \\
\text { relação a concorrência }\end{array}$} & melhor que a concorrência & 1,2 e 3 \\
\cline { 2 - 3 } & igual a concorrência & 4,5 e 6 \\
\cline { 2 - 3 } & pior que a concorrência & 7,8 e 9 \\
\hline
\end{tabular}

A avaliação dos indicadores de desempenho nestas duas dimensões (importância para o cliente e desempenho em relação a concorrência) quando colocadas na matriz de importância de desempenho permite definir uma prioridade de melhoria para cada um destes indicadores (figura 2). Esta matriz define quatro áreas de desempenho:

- Apropriada: nível de desempenho ideal para a empresa;

- Melhoria: nível de desempenho abaixo o limite da zona apropriada;

- Ação urgente: atingimento do desempenho está abaixo do que deveria ser;

- Excesso: atingimento do desempenho é muito melhor do que poderia parecer necessário.

Observe que o gráfico destaca o fato que um baixo desempenho num critério ganhador de pedido é muito mais grave que um baixo desempenho num critério menos importante. As áreas do gráfico procuram refletir a importância da combinação dos valores possíveis das duas dimensões dos indicadores de desempenho: Importância para o cliente e Desempenho em relação a concorrência

O uso desta ferramenta apresenta como vantagem a possibilidade de se comparar projetos que têm impactos diferentes em diferentes indicadores de desempenho. Assim, projetos que atuem sobre indicadores que estejam na zona de ação urgente são, segundo este modelo, mais importantes que os que têm impacto sobre os indicadores localizados a região de excesso.

Figura 2: Matriz de desempenho de Slack 


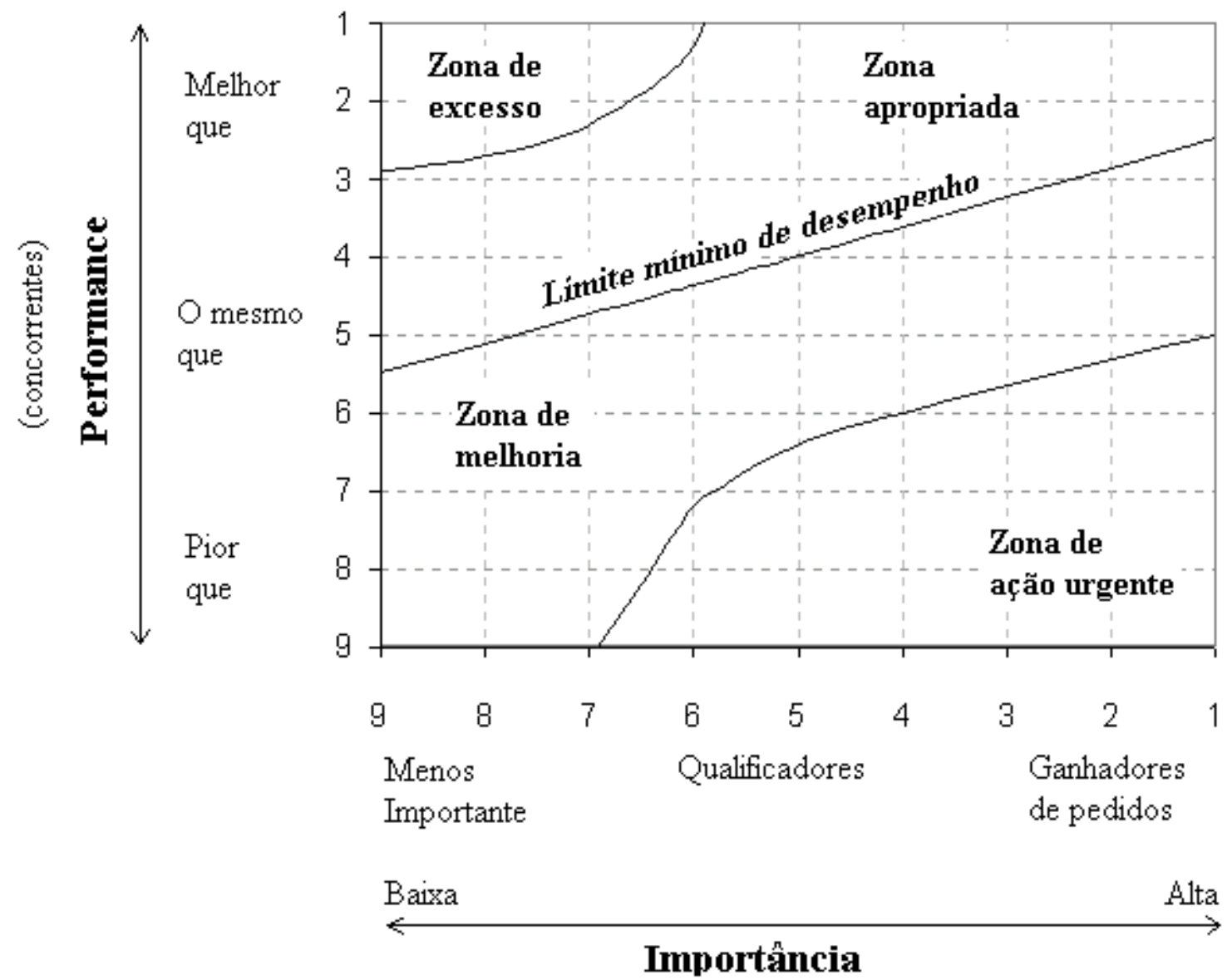

(clientes)

Fonte: Slack et all (A996)

\section{A proposta}

A incerteza neste trabalho se refere à probabilidade dos projetos terem impactos sobre os FCS de sucesso da organização. Para se realizar a estimativa da incerteza destes impactos da carteira, partes-se de estimativas individuais (de cada projeto) nos FCS da organização.

Para ilustrar o procedimento proposto, considere, a título de exemplo, uma organização que possui apenas 4 FCS. A tabela 1 e a figura 2 mostram a situação atual destes FCS. É bom lembrar que no uso feito aqui do modelo e GAP's os FCS são avaliados em duas dimensões: a importância dada pelo mercado e o desempenho em relação à concorrência. As avaliações estão numa escala onde valores menores são melhores que os maiores. Assim sendo, o FCS 1 é que apresenta uma situação mais preocupante. É o FCS de maior importância (ganhador de pedido) e possui um baixo desempenho em relação à concorrência. O FCS 2 também apresenta um desempenho baixo, assim como o FCS1, mas a importância dele é menor. Desta forma, estes dois FCS estão na região de “Urgência” no gráfico do modelo de GAP's (figura 2). Os FCS 3 e 4, que têm importância menor, se encontram na região de melhoria do modelo.

Tabela 1: Situação dos FCS 


\begin{tabular}{|l|l|l|}
\hline FCS & $\begin{array}{l}\text { Importância (dada pelo } \\
\text { mercado) }\end{array}$ & $\begin{array}{l}\text { Desempenho atual } \\
\text { relação à concorrência) }\end{array}$ \\
\hline 1 & 1,8 & 6,5 \\
\hline 2 & 3,5 & 6,5 \\
\hline 3 & 4,5 & 5,5 \\
\hline 4 & 6,5 & 7,3 \\
\hline
\end{tabular}

Figura 2: Situação dos FCS no Modelo de GAP's

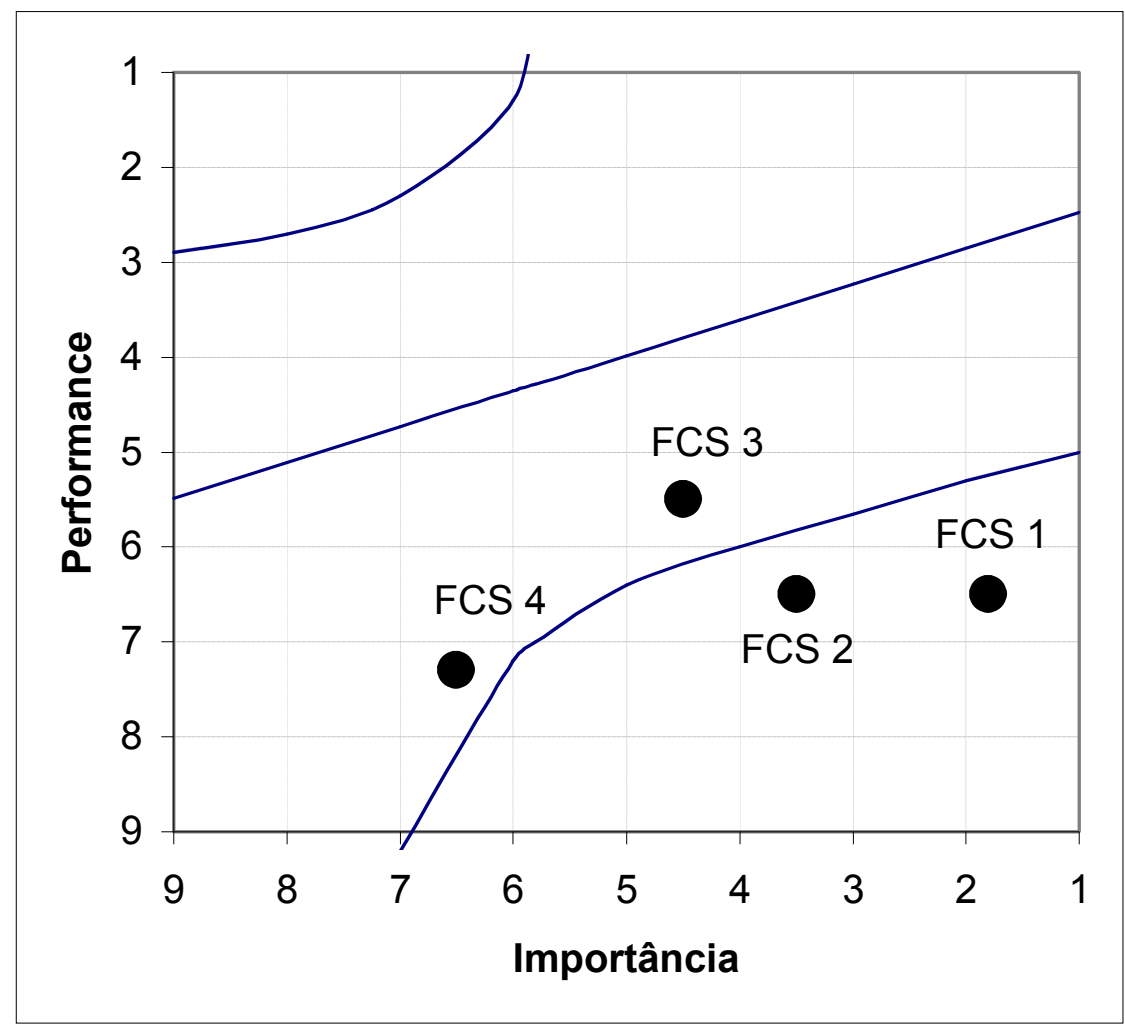

A tabela 2 mostra o impacto dos projetos de uma determinada carteira nestes FCS. Observe que para cada projeto são feitas três estimativas de impacto:

- a pessimista: refere-se ao pior caso possível, onde seriam obtidos os resultados mínimos possíveis com o projeto;

- a mais provável: refere-se a situação que tem mais chances de ocorrer e seus impactos sobre os FCS; e

- a otimista: refere-se ao melhor caso possível, onde os impactos mais significativos sobre os FCS seriam obtidos.

É fácil perceber que existe uma grande simplificação nestas estimativas, já que os impactos de um projeto em cada uma dos FCS não são, necessariamente, dependentes ou correlacionados.

Contudo, uma tripla estimativa de impacto representa um avanço em relação aos modelos encontrados na bibliografia da área. 
Tabela 2: Probabilidade de ocorrência dos possíveis resultados de cada projeto da carteira

\begin{tabular}{|c|c|c|c|c|}
\cline { 2 - 4 } \multicolumn{1}{c|}{} & \multicolumn{3}{c|}{ Resultados } & \multirow{2}{*}{ Tipo de Projeto } \\
\hline Projetos & Pessimista & Mais Provável & Otimista & \\
\hline 1 & $20,00 \%$ & $55,00 \%$ & $25,00 \%$ & Obrigatório \\
\hline 2 & $45,00 \%$ & $50,00 \%$ & $5,00 \%$ & Exploratório \\
\hline 3 & $35,00 \%$ & $55,00 \%$ & $10,00 \%$ & Exploratório \\
\hline 4 & $5,00 \%$ & $75,00 \%$ & $20,00 \%$ & Incremental \\
\hline 5 & $5,00 \%$ & $60,00 \%$ & $35,00 \%$ & Incremental \\
\hline 6 & $15,00 \%$ & $65,00 \%$ & $20,00 \%$ & Infra estrutura \\
\hline
\end{tabular}

Como existem seis projetos na carteira, e para casa projeto existem três desfechos distintos (o pessimista, o mais provável e o otimista), existe para este carteira $729\left(3^{6}\right)$ resultados possíveis para carteira. Estes resultados alternativos podem ser avaliados facilmente numa planilha eletrônica. Assim, o resultado mais otimista, apesar de para certos projetos terem uma probabilidade alta, o resultado mais otimista para a carteira (quando todos os projetos têm o desfecho mais otimista) é extremamente improvável (17,5 em um milhão).

Tabela 3: Impactos dos projetos sobre os FCS da organização

\begin{tabular}{|c|c|c|c|}
\cline { 2 - 4 } \multicolumn{1}{c|}{ FCS 1} & \multicolumn{3}{c|}{ Contribuição } \\
\hline Projetos & Pessimista & Mais Provável & Otimista \\
\hline 1 & $0,00 \%$ & $0,20 \%$ & $0,40 \%$ \\
\hline 2 & $0,00 \%$ & $1,00 \%$ & $7,00 \%$ \\
\hline 3 & $0,00 \%$ & $0,80 \%$ & $1,00 \%$ \\
\hline 4 & $1,00 \%$ & $3,00 \%$ & $4,00 \%$ \\
\hline 5 & $0,60 \%$ & $2,50 \%$ & $3,00 \%$ \\
\hline 6 & $0,20 \%$ & $0,50 \%$ & $1,00 \%$ \\
\hline
\end{tabular}

\begin{tabular}{|c|c|c|c|}
\cline { 2 - 4 } \multicolumn{1}{c|}{ FCS 2} & \multicolumn{3}{|c|}{ Contribuição } \\
\hline Projetos & Pessimista & Mais Provável & Otimista \\
\hline 1 & $0,00 \%$ & $0,20 \%$ & $0,30 \%$ \\
\hline 2 & $0,00 \%$ & $0,30 \%$ & $1,00 \%$ \\
\hline 3 & $0,00 \%$ & $4,00 \%$ & $7,00 \%$ \\
\hline 4 & $0,20 \%$ & $0,60 \%$ & $1,20 \%$ \\
\hline 5 & $1,20 \%$ & $2,30 \%$ & $3,60 \%$ \\
\hline 6 & $2,50 \%$ & $3,00 \%$ & $4,50 \%$ \\
\hline
\end{tabular}

\begin{tabular}{|c|c|c|c|}
\cline { 2 - 4 } \multicolumn{1}{c|}{ FCS 3} & \multicolumn{3}{|c|}{ Contribuição } \\
\hline Projetos & Pessimista & Mais Provável & Otimista \\
\hline 1 & $0,00 \%$ & $0,10 \%$ & $0,20 \%$ \\
\hline 2 & $0,00 \%$ & $0,40 \%$ & $1,00 \%$ \\
\hline 3 & $0,00 \%$ & $1,00 \%$ & $8,00 \%$ \\
\hline 4 & $1,00 \%$ & $3,00 \%$ & $4,00 \%$ \\
\hline 5 & $1,00 \%$ & $1,50 \%$ & $2,00 \%$ \\
\hline 6 & $3,00 \%$ & $6,00 \%$ & $8,00 \%$ \\
\hline
\end{tabular}

\begin{tabular}{|c|c|c|c|}
\cline { 2 - 4 } \multicolumn{1}{c|}{ FCS 4} & \multicolumn{3}{|c|}{ Contribuição } \\
\hline Projetos & Pessimista & Mais Provável & Otimista \\
\hline 1 & $0,00 \%$ & $0,20 \%$ & $0,30 \%$ \\
\hline 2 & $0,00 \%$ & $0,10 \%$ & $0,50 \%$ \\
\hline 3 & $0,00 \%$ & $0,10 \%$ & $0,50 \%$ \\
\hline 4 & $0,50 \%$ & $1,00 \%$ & $2,00 \%$ \\
\hline 5 & $2,00 \%$ & $2,50 \%$ & $3,00 \%$ \\
\hline 6 & $0,50 \%$ & $1,00 \%$ & $1,50 \%$ \\
\hline
\end{tabular}

A avaliação das diferentes combinações possíveis entre os projetos permite avaliar a probabilidade dos diferentes impactos que a carteira possa ter sobre os FCS. A representação deste impacto é feita através dos seguintes valores (Figura 2) expostos no gráfico de GAPs (Figura 3):

- Valor atual do FCS:

- Mínimo: refere-se ao impacto mínimo sobre o FCS, equivale a improvável situação na qual todos os projetos terminam da forma mais pessimista. 
- Primeiro quartil: a probabilidade de que o impacto sobre o FCS seja menor ou igual a este valor é $25 \%$

- Mediana: a probabilidade de que o impacto sobre o FCS seja menor ou igual a este valor é $50 \%$ (esta também é a probabilidade do impacto ser igual ou maior que este valor)

- Terceiro quartil: a probabilidade de que o impacto sobre o FCS seja maior ou igual a este valor é $25 \%$

- Máximo: refere-se ao impacto máximo sobre o FCS, equivale a improvável situação na qual todos os projetos terminam da forma mais otimista.

Tabela 4: Impactos da carteira sobre os FCS da organização

\begin{tabular}{|l|c|c|c|c|}
\hline \multirow{2}{*}{} & \multicolumn{4}{|c|}{ FCS } \\
\cline { 2 - 5 } & 1 & 2 & 3 & 4 \\
\hline Situação atual do FCS & 6,5 & 6,5 & 5,5 & 7,3 \\
\hline Mínimo & 6,0 & 5,5 & 4,5 & 6,5 \\
\hline Primeiro Quartil & 5,6 & 5,1 & 3,8 & 6,1 \\
\hline Mediana & 5,5 & 5,0 & 3,7 & 6,0 \\
\hline Terceiro Quartil & 5,4 & 4,8 & 3,5 & 5,3 \\
\hline Máximo & 4,8 & 4,0 & 2,7 & 5,0 \\
\hline
\end{tabular}

Figura 3: Representação do FCS no gráfico

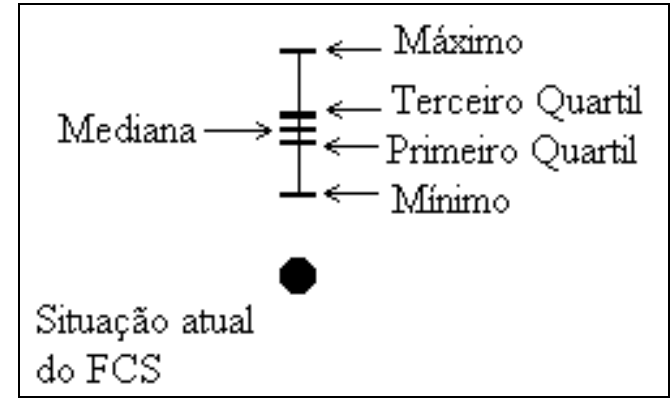

O impacto da carteira sobre os FCS pode ser então analisado através do modelo de GAPs (ver Figura 4). Observe que o impacto da carteira sobre o FCS 1 não fará com ele saia da zona de urgência em mais de $75 \%$ dos casos. Isto é, com esta composição da carteira de projetos, apesar da existência de impactos positivos sobre este FCS, eles, provavelmente, não serão suficientes para colocar o seu desempenho numa zona apropriada.

O FCS, 2 que está numa zona de urgência, com esta composição da carteira, passará para uma faixa de melhoria. Apesar da incerteza em relação aos impactos sobre o FCS 2, neste caso sabe-se, com certeza, das estimativas iniciais, em qual faixa do modelo de GAPs ele irá se colocar. Isto é, ele sairá da área de urgência e irá para área de melhoria (ver figura 4) Este tipo de resultado 
esperado é bastante interessante em termos qualitativos. Sabe-se que o FCS 2 deixará a faixa de urgência, mas mesmo assim ainda deverá alvo de ações futuras para a melhoria de seu desempenho.

Com relação ao FCS 3, a probabilidade dele sair da região de melhoria é de 75\% (Observe que o primeiro quartil está sobre a linha de desempenho mínimo). Talvez seja este o impacto mais significativo que se possa esperar desta carteira.

Quanto ao FCS 4, é certo que ele ainda continuará na região de melhoria, com esta composição da carteira.

Figura 4: Modelo de GAPs

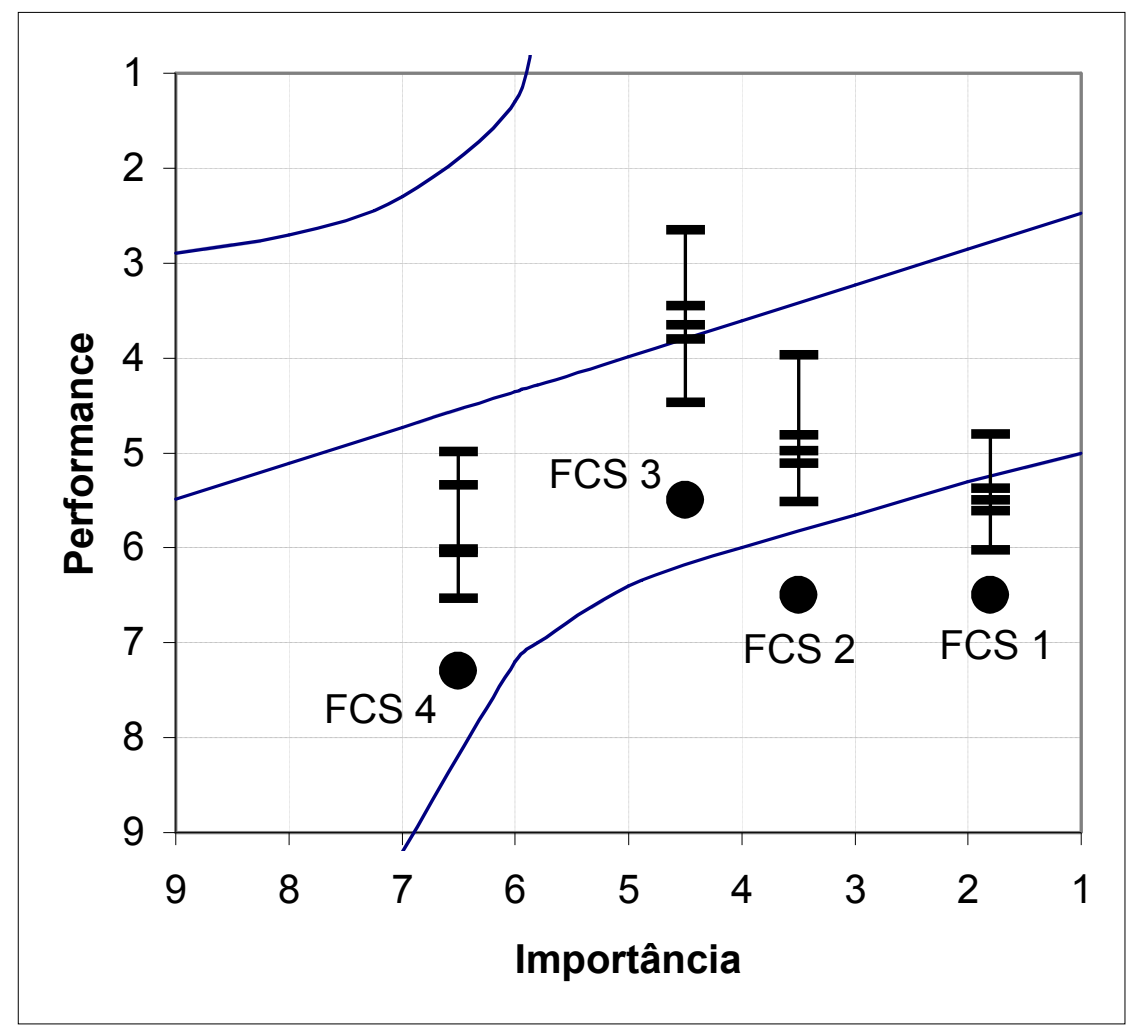

Assim, talvez o pior aspecto da carteira em análise seja o seu impacto sobre o FCS 1. A análise sobre como melhorar este impacto envolve a identificação dos projetos que menos contribuem para ele. Isto poderá ser feito através, por exemplo, de um gráfico como da figura 5.

O projeto 1 pouco contribui para este FCS, mas, sendo ele um projeto obrigatório, não existe a alternativa de não executá-lo. Poderia ser verificada a possibilidade uma eventual ampliação de seu escopo para que seu impacto potencial sobre este FCS seja maior.

O projeto 6 também possui um baixo impacto potencial sobre o FCS 1. Trata-se de um projeto de infra-estrutura cujos impactos diretos são normalmente baixos. Deve-se avaliar aqui duas alternativas: (i) ampliação de seu escopo, e a conseqüente ampliação de seu custo e incerteza; e (ii) 
substituição deste projeto por outro na carteira, eventualmente um projeto incremental. Neste último caso, os decisores devem lembrar que estarão trocando os benefícios potenciais futuros do projeto de infra-estrutura pelos benefícios imediatos de um projeto de natureza incremental.

Figura 5: Impacto dos projetos da carteira sobre o FCS 1

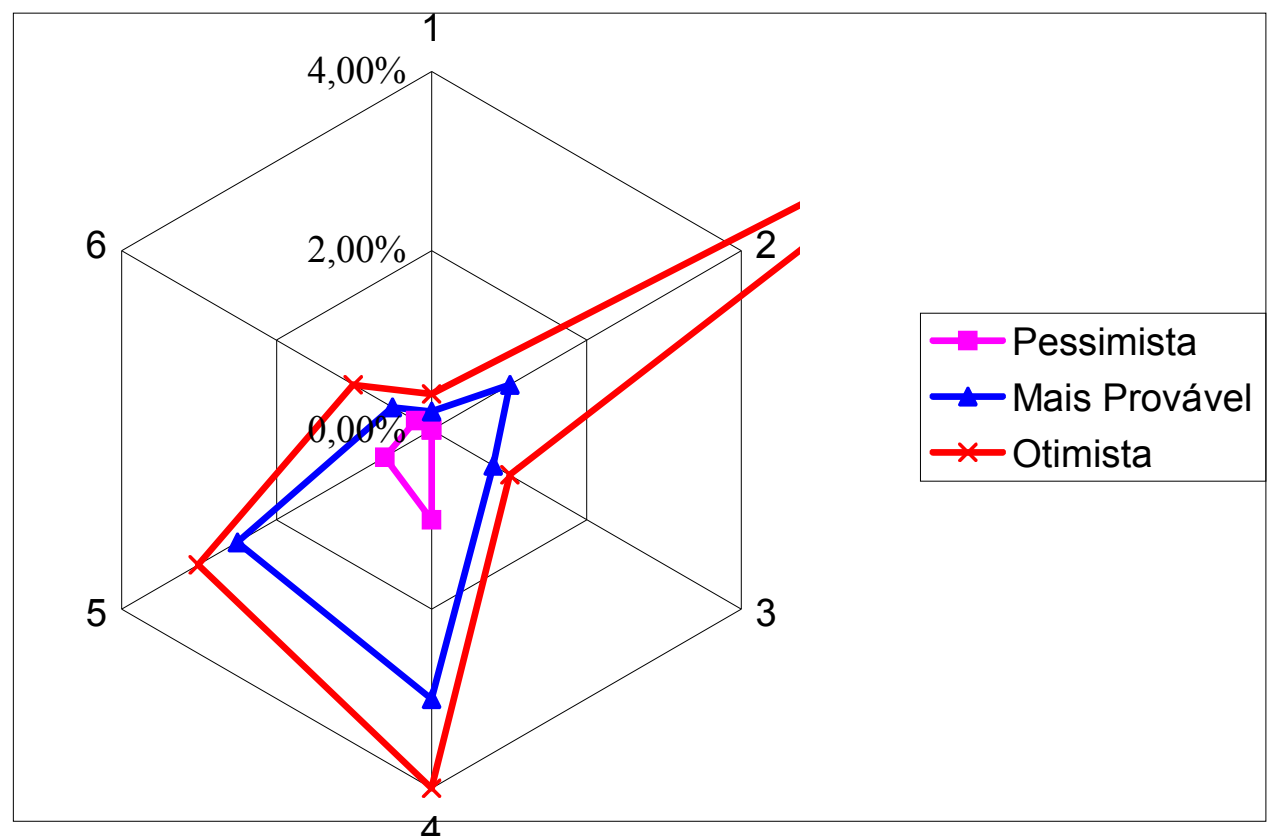

Os projetos 4 e 5 são incrementais e são eles que mais contribuem com o FCS 1, pelo menos em termos de uma menor incerteza dos impactos. Os projetos 2 e 3 são exploratórios. O projeto 2 tem, no caso mais otimista, um alto impacto sobre FCS 1, ao contrário do projeto 3. Neste caso, talvez, se dava considerar a possibilidade de substituição de um destes projetos (o projeto 3) por outro incremental, onde os benefícios imediatos seriam mais certos. Os decisores devem ter em mente que ao eliminar projetos exploratórios diminuem as chances de conseguir uma vantagem ampla e distintiva sobre a concorrência em troca de benefícios potenciais menores, porém mais certos no curto prazo. Note que o projeto 3 possui um grande impacto potencial (caso mais otimista) sobre o FCS 2 (principalmente) e o FCS 3.

Este tipo de análise, que leva a alteração da carteira, deve considerar o tamanho (custo) do projeto e as alternativas existentes. Isto é, ao se eliminar um projeto da carteira, qual(is) projeto(s) poderiam fazer parte da carteira, mantendo-se o orçamento total igual (ou quase igual)?

A análise da incerteza do portfólio pode, com os dados gerados, ser avaliada de forma integrada. Observe que, do ponto de vista da organização, o que interessa é o impacto da carteira de projetos na organização e não o de projetos individuais.

Contudo, a empresa aceitará um ou outro projeto de baixo desempenho se o conjunto de 
projetos, como um todo, tiver um desempenho satisfatório. A definição de "um desempenho satisfatório“ para a carteira de projetos é subjetiva, porém a organização, no processo de desenvolvimento de seu portfólio, deve adotar critérios para estimar a probabilidade de obtê-lo.

Para ilustrar, voltemos ao exemplo tratado anteriormente. Suponha que para a situação em questão, a carteira, para ser considerada um sucesso completo, deva fazer com que o FCS 1 tenha uma melhoria de pelo menos 12\%, o FCS 2 melhore em pelo menos $8 \%$, e que ou o FCS 3 melhore em $12 \%$ ou o FCS 4 melhore em $9 \%$.

Com os dados disponíveis é possível identificar em quais situações, e a probabilidade de ocorrência de cada situação, na qual a carteira consegue tal desempenho. A figura 5 ilustra esta análise. É possível, portanto, com os dados disponíveis, estimar que a carteira tem uma probalidade de apenas 3,4\% de conseguir esta performance (sucesso completo). Contudo, se o desempenho da carteira, para ser considerado como bom - sucesso satisfatório - os fatores críticos devem atingir níveis mais baixos, como, por exemplo, 7\% para FCS 1, 6\% para o FCS 2, 3\% para o FCS 3 e 5\% para o FCS 4, a carteira tem uma probabilidade de 76,2\% para conseguir este desempenho (sucesso satisfatório).

Assim , a partir de uma definição de desempenho através da melhoria dos FCS, é possível a avaliar a probabilidade de uma determinada carteira de projeto atingir diferentes níveis de desempenho.

\section{Considerações Finais}

O artigo apresentou uma proposta para avaliação da incerteza da carteira de projetos de desenvolvimento. Ao contrário da maioria dos trabalhos sobre incerteza de projetos, este procura avaliar a incerteza associada a um conjunto de projetos e não a projetos individuais. A incerteza associada a cada projeto é um dado de entrada do modelo. Os resultados sugerem que é possível, através da proposta apresentada, criar uma visão mais realista dos possíveis impactos de uma carteira em particular.

Uma limitação da proposta é que ela não leva em conta o aspecto temporal dos impactos dos projetos que compõem a carteira. Os projetos de uma carteira têm durações variadas, o que faz com que o impacto da carteira não se manifeste, em todo seu potencial, em único momento. Além disto, os impactos de um projeto também não se manifestam todos ao mesmo tempo. Esta situação é particularmente freqüente em projetos de infra-estrutura. Assim os impactos de um projeto sobre os indicadores de desempenho de uma organização é uma função do tempo e, portanto, da carteira de 
projetos também.

Esta limitação da proposta, sugere um possível desdobramento deste trabalho acrescentando a variável tempo ao procedimento proposto.

\begin{abstract}
The methods for building a project portfolio used to emphasize the importance of uncertainties as one important variable of this process. Usually, evaluation of uncertainties is performed for each project individually, as much for the uncertainties about the resources needed as well as for the uncertainties related to the results to be reached.

The "manager of project managers" is responsible for all projects (and their managers) and accepts to assume some degree of individual failure in some of the projects under his/her responsibility, but his/her ultimate concern is about the general result of the whole set of projects under development. Thus, for building a projects portfolio is very important an evaluation of the uncertainty of the possible alternatives of projects portfolio - uncertainty associated to a particular group of projects.

This paper continues the discussion of the proposal presented in the paper of Laurindo and Moraes (2004) for composing an IT development projects portfolio, emphasizing the question of projects uncertainties.

The use of the critical success factors - CSF (ROCKART, 1979) is substituted by the Gaps Analysis (SLACK et all, 1996). Besides, the estimate of the uncertainty related to the contribution of each project to the organizational performance indicators is performed through three different scenarios: Pessimist, More probable and Optimist:

The evaluation of the different possible combinations of the projects allows estimating the probability of the different impacts that the portfolio can have on the organizational performance indicators.
\end{abstract}

Keywords: Project Uncertainties, Portfolio Uncertainties, Portfolio Management

\title{
Referências Bibliográficas
}

ARCHIBALD, R. D. Managing high technology programs and projects. New York, John Willey, 1976,278 p. BACCARINI, D. The Concept of project complexity - a review. International Journal of Project Management vol. 14, no. 4, pp 201-204, 1996

cross ${ }^{\text {ref }}$

MORAES, R. O. Planejamento, Programação e Controle de Projetos de Software. Dissertação de mestrado submetida à Universidade Paulista. São Paulo, 1999.

MORAeS, R. O. ; LAURINDO, F. J. B. Managing IT Projects Portfolio in a Brazilian Company. In 11 th International Annual Euroma Conference. France: INSEAD. 27-30 June 2004

MORAeS, R. O. ; LAURIndo, F. J. B. Seleção de Projetos de TI: Gestão do Portfólio de Aplicações. In LAURINDO, F. J. B. ; ROTONDARO, R. G. Gestão Integrada de Processos e da Tecnologia da Informação. São Paulo: Ed. Atlas, 2006

ROCKART, J. F. Chief Executives define their own data needs. Harvard Business Review, v. 57, n. 2, p. 81-92, Mar./Apr. 1979.

SHENHAR, Aaron J. et al Refining the search for project success factors: a multivariate, typological approach. R 
\& D Management; Mar 2002, Vol. 32 Issue 2, p111, 16p

SLACK, Niegel et al. Administração da Produção. São Paulo: Ed Atlas, 1996

SOUDER, W. E. Selecting Projects that Maximize Profits IN CLELAND, D. I. \& KING, W. R. Handbook of Project Management. New York: McGraw Hill, 1983

Nome completo: Renato de Oliveira Moraes

Filiação institucional: Universidade Federal de Ouro Preto

Departamento: Departamento de Ciências Exatas e Aplicadas

Função ou cargo ocupado: Professor Adjunto

Endereço completo: Av Afonso Treze, 821, ap. 1.101 - Gutierrez - Belo Horizonte - MG

Cep 30.430-170

Telefones para contato: (31) 3334-4977

e-mail: renato@decea.ufop.br renato_om@hotmail.com

Nome completo: Fernando José Barbin Laurindo

Filiação institucional: Universidade de São Paulo

Departamento: Departamento de Engenharia de Produção da Escola Politécnica

Função ou cargo ocupado: Professor Associado

Endereço completo: Rua José Maria Lisboa, 368 ap. 51 - Jardim Paulista - São Paulo - SP

CEP 01423-000

Telefone para contato: (11) 3091-5363 ramal 454

e-mail: fjblau@usp.br

Nome completo: Priscila Maria Santiago pereira

Filiação institucional: Universidade Federal de Minas Gerais

Departamento: Departamento de Engenharia Sanitária e Ambiental

Função ou cargo ocupado: aluna de pós graduação

Endereço completo: Av Afonso Treze, 821, ap. 1.101 - Gutierrez - Belo Horizonte - MG

Cep 30.430-170

Telefones para contato: (31) 3334-4977

e-mail:prisicla.santiago@yahoo.com.br

Recebido para publicação em:04/02/2008

Aceito para publicação em: 07/03/2008 